\title{
Magnésium et effort d'endurance chez le poney
}

\author{
R. WOLTER, J.P. VALETTE, J.M. MARION \\ I.N.R.A., Laboratoire de Nutrition et Alimentation, Ecole Nationale Vétérinaire d'Alfort \\ 7, avenue du Général-de-Gaulle, F 94704 Maisons-Alfort Cedex
}

\begin{abstract}
Résumé
Chez des poneys régulièrement entraînés à des efforts sportifs d'endurance, et recevant des régimes à 3 ou 20 p. 100 de matières grasses (par adjonction soit de saindoux, soit d'un mélange de saindoux, de graisse de coprah et d'huile de tournesol), un taux magnésique de 0,11 p. 100, correspondant à peu près aux recommandations du NRC (1978) entraîne une faible digestibilité apparente du magnésium (surtout avec le régime enrichi en saindoux) et une médiocre stabilité de la magnésie (en particulier lors de travail soutenu).

Une supplémentation pour atteindre environ 0,18 p. 100 de magnésium dans la ration apparaît favorable à la digestibilité apparente du magnésium et au meilleur maintien de la magnésémie. Elle semble devoir être conseillée pour le cheval de sport soumis à des efforts, d'autant plus que le régime est complémenté en graisses à acides gras et saturés, que la lipolyse est plus intense, et que la sudation est plus abondante.
\end{abstract}

Mots clés : Magnésium, effort d'endurance, régimes hyperlipidiques, digestibilité du magnésium, hypomagnésémie à l'effort.

\section{Introduction}

Chez le cheval, la digestibilité apparente du magnésium atteint en moyenne 50 p. 100, c'est-à-dire bien plus que chez les ruminants. Elle est diminuée par les excès de phosphates (Hintz \& Schryver, 1972) et même de calcium (Hintz \& Schryver, 1973). Selon Hintz \& Schryver (1972), l'augmentation du niveau magnésique de la ration de 0,10 p. 100 à 0,31 p. 100 se traduit par une élévation des digestibilités apparente et réelle du magnésium, alors même que si l'on passe de 0,31 p. 100 à 0,86 p. 100 , il n'y a pas de répercussion significative. Il faut préciser cependant que le bas niveau n'est assuré que par le magnésium intrinsèque à la ration de base tandis que les deux niveaux plus élevés procèdent d'une addition d'oxyde de magnésium. Or, celui-ci, ainsi que quelques autres sources minérales (carbonate et sulfate de magnésium) est signalé comme hautement disponible au niveau intestinal (GERKEN \& Fontenot, 1967 ; WaLSh \& Harrington, 1973). L'absorption se déroule essentiellement dans l'intestin grêle (pour 46 à 56 p. 100 de magnésium ingéré), surtout en zone iléale; l'absorption dans le gros intestin ne dépasserait pas 5 p. 100 (Hintz \& Schryver, 1972). L'augmentation de l'apport magnésique se traduit par 
une élévation nette et rapide de la magnésémie (HinTz \& SCHRyver, 1973 ; Walsh \& HARRINGTON, 1973 ; HARRINGTON, 1974).

D'après le « National Research Council» (1978), les besoins alimentaires du cheval se situent vers $1000 \mathrm{ppm}(0,1 \mathrm{p}$. 100) de magnésium dans la ration (soit environ $20 \mathrm{mg} / \mathrm{kg} \mathrm{PV}$ ). Cette norme approche les estimations de HinTz \& Schryver (1972) et de Meyer (1979) : $15 \mathrm{mg} / \mathrm{kg} \mathrm{PV} / \mathrm{j}$ (1,5 g/100 kg PV). Mais chez les chevaux soumis à un travail musculaire soutenu, ces besoins peuvent être nettement accrus afin d'éviter une baisse sensible de la magnésémie : d'une part, la sudation provoque une perte magnésique de l'ordre de $12 \mathrm{mg}$ par $100 \mathrm{ml}$ de sueur (Costrll, 1977 ; Lucke \& HaLl, 1980), d'autre part, l'effort prolongé entraîne une lipolyse, laquelle expose à une fixation du magnésium sur les membranes des cellules adipeuses (RAYssiguier \& LARvor, 1976). Toutefois, la décroissance de la magnésémie n'est retrouvée que de façon extrêmement modérée par CARLson \& Mansmann (1974). Une légère élévation de la concentration magnésique initiale est même observée quelquefois (SNow et al., 1982 ; Grosskopf et al., 1983).

Ces différences de résultats concernant l'évolution de la magnésémie en fonction du travail musculaire pourraient dériver des variations du degré d'hémoconcentration (SNOw et al., 1982), celle-ci traduisant d'abord la déplétion de la rate et surtout la déshydratation liée à la sudation qui dépend de l'intensité de l'effort, de sa durée, de l'état d'entraînement du cheval, des conditions atmosphériques... Malgré tout, une hypomagnésémie est bien constatée chez le cheval (LAPRAS, 1979 ; Huguet et al., 1981) et chez le chien (Grandjean, 1983).

Ce danger d'hypomagnésémie chez le cheval entraîné aux courses d'endurance pourrait être encore renforcé par le recours à des régimes hyperlipidiques dont nous avons montré l'intérêt (Wolter \& VAletTe, 1986).

Le but du présent essai est donc d'étudier l'influence d'une supplémentation en magnésium sur la digestibilité de régimes hyperlipidiques et sur l'aptitude sportive des poneys recevant ces régimes.

\section{Matériel et méthodes}

\section{A. Animaux et régimes alimentaires}

Cinq poneys (mâles, adultes) reçoivent 5 régimes selon une répartition en carré latin en deux repas à 8 h et à 17 h (lors des épreuves, ils restent à jeûn et reçoivent un repas 2 heures après la fin de celles-ci).

La composition des régimes est indiquée dans le tableau 1 :

- T, régime témoin à 3 p. 100 de matières grasses (aliment complet du commerce à base de foin broyé, son, orge, avoine, caroube, tourteau de soja et complément minéral vitaminé) ;

- $\mathrm{T}^{+}$, régime témoin complémenté en magnésium ;

- $\mathrm{S}$, régime à 20 p. 100 de matières grasses, apportées par du saindoux (teneur élevée en acides gras longs saturés); 
- SH, 20 p. 100 de matières grasses apportées par un mélange comportant $1 / 2$ de saindoux, 1/4 d'huile de tournesol (teneur élevée en acides gras longs polyinsaturés) et $1 / 4$ de graisse de coprah (teneur élevée en acides gras de longueur moyenne ;

- $\mathrm{SH}^{+}$, le régime précédent complémenté en magnésium.

\section{TABleaU 1}

Composition des régimes expérimentaux.

Composition of experimental diets.

\begin{tabular}{|c|c|c|c|}
\hline & $\mathrm{T}\left(\mathrm{T}^{+}\right)^{*}$ & $S$ & $\mathrm{SH}\left(\mathrm{SH}^{+}\right)^{*}$ \\
\hline Aliment complet du commerce & 100 & 82 & 82 \\
\hline Commercial diet & & & \\
\hline Saindoux - Lard & 0 & 18 & 9 \\
\hline Huile de tournesol - Sunflower oil & 0 & 0 & 4,5 \\
\hline Huile de coprah - Coconut oil .... & 0 & 0 & 4,5 \\
\hline Matière sèche - Dry matter ......... & 89,0 & 91,0 & 91,0 \\
\hline & & & 111 \\
\hline 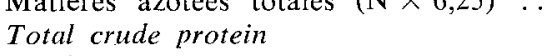 & 13,5 & 11,3 & 11,1 \\
\hline Matières grasses - Fat .. & 3,5 & 22,7 & 22,7 \\
\hline Matières minérales - Minerals & 11,2 & 9,0 & 9,0 \\
\hline Calcium $\ldots \ldots \ldots \ldots \ldots \ldots$ & 1,15 & 0,90 & 0,90 \\
\hline Phosphore - Phosphorus & 0,55 & 0,45 & 0,45 \\
\hline Magnésium $\ldots \ldots \ldots \ldots \ldots$ & 0,13 & 0,11 & 0,11 \\
\hline Cellulose brute - Crude fibre & 20,7 & 16,6 & 16,6 \\
\hline Extractif non azoté - Protein-free extract & 49,2 & 39,1 & 39,3 \\
\hline
\end{tabular}

* Les régimes $\mathrm{T}^{+}$et $\mathrm{SH}^{+}$ont été enrichis en magnésium.

Diets $\mathrm{T}^{+}$and $\mathrm{SH}^{+}$were supplemented with magnesium.

Les régimes $\mathrm{T}^{+}$et $\mathrm{SH}^{+}$sont complémentés avec 1,8 $\mathrm{g}$ de magnésium par animal et par jour sous forme de chlorure de magnésium anhydre associé à du glycocolle. L'apport quotidien est ainsi de $4,3 \mathrm{~g} / \mathrm{j}$ en moyenne alors que le NRC (1978) recommande entre $2,1 \mathrm{~g}$ et $3,4 \mathrm{~g}$ pour des animaux pesant environ $200 \mathrm{~kg}$ (tabl. 2).

L'énergie brute des aliments (en $\mathrm{kcal} / \mathrm{kg}$ MS) a été calculée par la formule de SCHIEMANN et al (1971) :

$$
\mathrm{EB}=5,72 \mathrm{MAT}+9,50 \mathrm{MG}+4,79 \mathrm{CB}+4,17 \mathrm{ENA}+\Delta
$$

La digestibilité de l'énergie $(\mathrm{dE})$, calculée précédemment sur des régimes analogues (VALETTE, 1984), selon la formule : $\mathrm{dE}=$ Energie disgestible ED/Energie brute (EB), avec $\mathrm{ED}=5,65 \mathrm{MAd}+9,45 \mathrm{MGd}+4,15$ (CBd + ENAd) où MAd, $\mathrm{MGd}, \mathrm{CBd}$ et ENAd représentent les matières azotées digestibles, les matières grasses digestibles, la cellulose brute digestible et l'extractif non azoté digestible, donne des valeurs de 0,76 pour le régime témoin et 0,72 pour les régimes gras; ce qui 
correspond respectivement à 3300 et $3900 \mathrm{kcal}$ ED $/ \mathrm{kg}$ MS. Cette digestibilité de l'énergie est corrélée à la digestibilité de la matière organique par la relation suivante :

$$
\mathrm{dE}=0,90 \mathrm{dMO}+0,110(\mathrm{r} 7 \mathrm{ddl}=0,951 \mathrm{~s} \text { à } 1 \mathrm{p} .1000)
$$

relation que l'on peut rapprocher de l'équation de NeHring et al. (1963) pour les bovins :

$$
\mathrm{dE}=\mathrm{dMO}-0,015
$$

Les rations distribuées exprimées en $\mathrm{g}$ de $\mathrm{MS}$ par $\mathrm{kg}$ de poids métabolique ( $P V^{0,75}$ ) sont alors de 47 pour le régime témoin et de 40 pour les régimes hyperlipidiques, afin de fournir les $155 \mathrm{kcal} \mathrm{ED} / \mathrm{kg} \mathrm{PV}^{0, \pi 5}$ (NRC, 1978) nécessaires à la stricte couverture des besoins d'entretien.

\section{B. Protocole expérimental}

Après 15 jours d'adaptation à chaque régime, une prise de sang est effectuée ( 2 heures après le repas du matin) et commencent les mesures de digestibilité par récolte totale des fèces pendant 5 jours (WoLTER et al., 1982). Les prélèvements de fèces sont soumis aux analyses classiques (VALETTE, 1984) ; en particulier, le magnésium est dosé par spectrométrie d'absorption atomique après reprise des cendres dans un tampon lanthane.

A la suite des mesures de digestibilité, les poneys sont remis à l'entraînement pendant une semaine ( 3 séances de 1 heure au trot léger) puis sont soumis à une épreuve sportive test qui intervient donc après une adaptation de 4 semaines à chacun des régimes.

Cette épreuve sportive standard imposée aux animaux à jeûn est reproduite au moyen d'une longe électrique selon un protocole déjà décrit (WoLTER et al., 1984). Elle donne lieu à des prélèvements sanguins par cathétérisme jugulaire de façon renouvelée à intervalles prédéterminés afin de suivre l'évolution des paramètres sanguins, notamment de la magnésémie $(0,30,120 \mathrm{mn}$, temps final variable, $30 \mathrm{mn}$ et $120 \mathrm{mn}$ après l'arrêt de l'épreuve).

Les résultats sont soumis à différents tests statistiques (SchWARTZ, 1969 ; LELLOUCH \& LAZAR, 1974).

\section{Résultats}

Les résultats des mesures de digestibilité du magnésium sont récapitulés dans le tableau 2.

L'analyse de variance en carré latin fait ressortir un effet «adjonction de magnésium » significatif à 1 p. 1000 , la digestibilité du magnésium des régimes $\mathrm{T}^{+}$ et $\mathrm{SH}^{+}$étant supérieure à celle des régimes $\mathrm{T}$ et $\mathrm{SH}$, eux-mêmes supérieurs au régime $\mathrm{S}$.

La magnésémie à jeûn (valeur de base) est de 725 micromoles/1 pour les régimes non supplémentés en magnésium et de 780 micromoles/1 pour les régimes supplémentés (valeurs non significativement différentes). 


\section{TABLEAU 2}

Digestibilité apparente (en p. 100) du magnésium* $(m+t s / \sqrt{ } n)$

(s étant l'écart-type estimé).

Apparent digestibility of magnesium (as p. 100) ( $s$ : estimated standard deviation).

\begin{tabular}{|c|c|c|c|c|c|}
\hline \multicolumn{6}{|c|}{ Régimes - Diets } \\
\hline $\begin{array}{l}\text { Mg ingéré } \\
(e n g / A 1 / j)\end{array}$ & $\mathbf{T}$ & $\mathrm{T}^{+}$ & SH & $\mathrm{SH}^{+}$ & $S$ \\
\hline $\begin{array}{l}M g \text { intake } \\
(\mathrm{g} / \text { diet } / d)\end{array}$ & 2,6 & 4,4 & 2,2 & 4,0 & 2,2 \\
\hline $\begin{array}{l}\text { CUD } \\
(\text { p. } 100)\end{array}$ & $14,0 \pm 13,5$ & $31,8 \pm 15,5$ & $21,4 \pm 14,9$ & $31,8 \pm 14,5$ & $0,6 \pm 20,0$ \\
\hline $\begin{array}{c}\text { Apparent digestibility } \\
(p .1 C 0)\end{array}$ & $\mathrm{a}$ & $\mathrm{b}$ & $\mathbf{a}$ & b & $c$ \\
\hline \multicolumn{6}{|c|}{$\begin{array}{l}\text { Les chiffres non affectés d'une même lettre diffèrent significativement au seuil de } 5 \text { p. } 100 \\
\text { Valtues with unlike letters are significantly different }(P<0.05) \text {. }\end{array}$} \\
\hline
\end{tabular}

Par ailleurs, les valeurs à jeûn (tous régimes confondus) sont de 745 ! moles $/ 1$ alors qu'elles sont de 825 umoles/l après repas (significatif à 1 p. 100).

$\mathrm{Au}$ cours de l'épreuve-test de type endurance qui dure environ 3 heures trente (fig. 1), la baisse de la magnésémie atteint en moyenne 11 p. 100 ; elle est plus accentuée avec les régimes témoins : $\mathrm{T}:-19 \mathrm{p} .100 ; \mathrm{T}^{+}=-14 \mathrm{p} .100$ (avec $\mathrm{F}_{12}^{4}=3,60$ significatifs à 5 p. 100 ), qu'avec les régimes gras : $\mathrm{S}:-6$ p. $100 ; \mathrm{SH}$ : -8 p. $100 ; \mathrm{SH}^{+}:-4$ p. 100 ; par contre, elle reste de même ampleur selon que la ration est supplémentée $(-9$ p. 100) ou non $(-11$ p. 100) en magnésium.

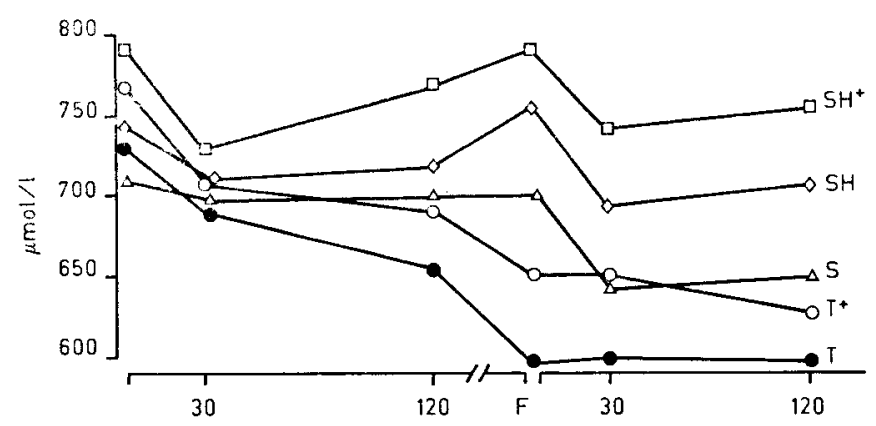

FIG. 1

Evolution de la magnésémie au cours des épreuves. Variation of magnesemia during effort. 
Par ailleurs, bien que soumis à un entraînement tri-hebdomadaire avec, tous les mois, une épreuve pouvant durer 4 heures, les animaux n'ont pas eu de variations significatives de poids sur la période expérimentale de 5 mois.

\section{Discussion}

\section{A. Digestibilité apparente du magnésium}

L'apport de magnésium par la ration de base apparaît assez bas : environ $2,5 \mathrm{~g} /$ poney $/ \mathrm{j}$, alors que le N.R.C. (1978) recommande au minimum $1,5 \mathrm{~g} / 100 \mathrm{~kg}$ $\mathrm{PV} / \mathrm{j}$, soit $3 \mathrm{~g} / \mathrm{j}$ pour des sujets d'environ $200 \mathrm{~kg}$. La faible digestibilité apparente du magnésium résulterait alors d'abord et surtout de la modeste disponibilité du magnésium contenu dans les matières premières par rapport aux sources minérales (GERKEN \& Fontenot, 1967 ; WALSh \& HARrIngton, 1973); elle pourrait aussi être amoindrie par la relative fixité de la perte endogène fécale.

La complémentation magnésique, à raison de $1,8 \mathrm{~g} /$ poney $/ \mathrm{j}$, permettant donc d'atteindre une fourniture totale de $4,3 \mathrm{~g} /$ poney/j, assure une large couverture des besoins calculés. Réalisée sous la forme d'un chélate glycocolle-magnésium hautement assimilable, elle contribue à augmenter sensiblement la digestibilité apparente du magnésium, déjà par cet aspect qualitatif, et éventuellement par son effet quantitatif conduisant à minimiser le retentissement de la perte endogène fécale.

L'adjonction de saindoux tend à réduire la digestibilité apparente du magnésium de la ration de base, vraisemblablement à cause de la formation de savons insolubles avec les acides gras saturés (RAYSSIGUIER, 1981) ; toutefois, l'absence d'un régime $\mathrm{S}^{+}$ (enrichi à la fois en saindoux et en magnésium) retire la possibilité de préciser une éventuelle interaction négative entre graisses animales et digestibilité du magnésium. Par contre, le mélange de saindoux-graisse de coprah-huile de tournesol n'a pas d'influence négative sur la digestibilité apparente du magnésium, alors même que sa propre digestibilité est meilleure que celle du saindoux, sans doute grâce à une meilleure émulsification intestinale.

\section{B. Variations de la magnésémie}

L'hypomagnésémie traduit rapidement un défaut d'apport et/ou d'utilisation digestive du magnésium (HINTz \& Schryver, 1973), ou encore une captation exagérée de celui-ci par le tissu adipeux. Ainsi, elle menace avec le régime de base $(610$ umoles $/ 1$ au lieu de $800 \mu$ moles/1 en moyenne). Elle est renforcée par l'incorporation alimentaire de saindoux. Elle est aggravée encore par le travail musculaire, comme par toute cause de lipolyse tissulaire qui provoque une fixation des ions $\mathrm{Mg}^{++}$par les adipocytes (Elliot \& Rizach, 1974) ; d'ailleurs, dans le cas présent, elle apparaît corrélée avec la sévérité de l'épreuve sportive (fig. 1).

Cette hypomagnésémie risque alors de se manifester par de l'anorexie, des tremblements musculaires, des raideurs et même des tétanies, comme à la suite d'un jeûne prolongé à l'origine d'un rapide amaigrissement, tel que cela a été observé dans notre laboratoire (RICAUX, 1982). Ainsi, elle s'apparente bien à la tétanie d'herbage 
des ruminants (LARVor \& VIOLETTE, 1969 ; LARVor \& RAYSSIGuier, 1978), qui est également favorisée par toute cause de lipolyse intense (RAYssiguiER, 1977). Elle est aussi rapidement corrigée par une complémentation magnésique (MARION, 1984). Par contre, elle peut resurgir dès l'arrêt de cette complémentation magnésique, prouvant que celle-ci a peu d'effet rémanent.

En outre, dans une autre partie de cet essai, rapportée par ailleurs, nous avons mis en évidence que la complémentation magnésique, chez des poneys soumis à un entraînement d'endurance sportive et recevant un régime hypergras, limite l'augmentation des taux d'acides gras libres plasmatiques à 255 p. 100 au lieu de 355 p. 100, alors que son effet est inverse avec un régime témoin pauvre en lipides (Wolter \& VALETTE, 1986).

En conclusion, un aliment complet usuel dosant 0,11 p. 100 de magnésium semble trop peu pourvu, si on en juge par la faible digestibilité apparente du magnésium et le bas niveau de la magnésémie ; toutefois, seules des mesures du bilan magnésique permettraient de définir un véritable état de carence et d'approcher la connaissance des besoins. Celui-ci ne pourrait qu'être renforcé par l'adjonction alimentaire de graisses riches en acides gras saturés qui tendent à réduire la digestibilité apparente du magnésium. En outre, le travail musculaire de longue durée, de type aérobie, contribue à abaisser directement la magnésémie, parallèlement à l'activation de la lipolyse tissulaire.

Il en résulte que les régimes hypergras, dont nous avons vérifié par ailleurs l'intérêt pour améliorer les performances sportives d'endurance chez le poney comme chez le chien, méritent d'être systématiquement complémentés en magnésium. Ainsi, dans cet essai, l'apport supplémentaire de 1,8 $\mathrm{g}$ de magnésium par poney et par jour, faisant passer le taux magnésique de la ration à 0,18 p. 100 environ, réhausse la digestibilité apparente du magnésium et assure une plus grande stabilité de la magnésémie chez les poneys entraînés à des efforts longs et recevant un régime à 24 p. 100 de matières grasses.

Dans ces conditions, la recommandation du NRC (1978) à 0,1 p. 100 de magnésium dans la ration du cheval risque d'être nettement insuffisante pour prévenir toute hypomagnésémie qui peut compromettre le bon contrôle de l'excitabilité neuromusculaire et même l'utilisation métabolique des acides gras. Une élévation sensible de cette norme apparaîtrait donc justifiée chez le cheval de sport, surtout en effort d'endurance et d'autant plus que la sudation est plus abondante.

Reçu en décembre 1985.

Accepté en juin 1986.

\section{Summary \\ Magnesium and endurance effort in ponies}

Ponies regularly trained to endurance rides were given diets (table 1) including 3 or 20 p. 100 fat (by addition of lard or of a mixture of lard and vegetable oils) and 0.11 p. 100 magnesium according to NRC recommendations (1978). 
Apparent digestibility of magnesium (table 2) was low (especially with the lard supplemented diet) and stability of magnesemia (fig. 1) was poor (particularly under sustained effort).

Supplementation of diets up to 0,18 p. 100 magnesium improved the apparent digestibility of magnesium and stability of magnesemia. This level of magnesium should be recommended for sporting horses during training, especially when their diets are supplemented with saturated long chain fatty acids, when lipolysis is intense and when sudation is abundant.

Key words : Magnesium, endurance effort, hyperlipidic diets, magnesium digestibility, hypomagnesemia during effort.

\section{Références bibliographiques}

Carlson G.P., Mansmann R.A., 1974. Serum electrolyte and plasma protein alterations in horses used in endurance rides. J. Am. Vet. Med. Ass. ; 165, 262-264.

Costill D.L., 1977. Sweating : its composition and effects on body fluids. N.Y. Acad. Sci.; 301, $160-174$.

ElliotT D.A., RizaCH M.A., 1974. Epinephrin and adrenocorticotropic hormonestimulated magnesium accumulation in adipocytes and their plasma membranes. J. Biol. Chem., 249, 3895-3990.

Gerken H.J., Fontenot J.P., 1967. Availability and utilisation of magnesium from dolomitic limestone and magnesium oxide in steers. J. Anim. Sci., 26, 1404-1408.

Grandjean D., 1983. Nutrition hyperlipidique et aptitudes sportives à l'effort d'endurance chez le chien. Etude des paramètres physiologiques, plasmatiques et hématologiques. Thèse Doct. $3^{\mathrm{e}}$ Cycle. Université Paris VI, $376 \mathrm{p}$.

Grosskopf J.F.W., Rensbure Van J.J., Bertschinger H.J., 1983. Haematology and blood biochemistry of horses during a $210 \mathrm{~km}$ endurance ride, pp. 416-424. Equine Exercice Physiology. Snow D.H., Persons S.G.B., Rose R.J., Cambridge ed.

Harrington D.D., 1974. Pathologic features of magnesium deficiency in young horses fed purified rations. Am. J. Vet. Res., 35, 503-513.

Hintz H.F., Schryver H.F., 1972. Magnesium in the horse. J. Anim. Sci., 35, 755-759.

Hintz H.F., Schryver H.F., 1973. Magnesium, calcium and phosphorus metabolism in ponies fed varying levels of magnesium. J. Anim. Sci., 37, 927-930.

Huguet J.M., Braun J.P., Benard P., Burgat-Sacaze V., Rico G., 1981. Sémiologie de la forme du cheval de sport dans les courses de vitesse (1). Rec. Méd. Vét., 157, 407-413.

Lapras M., 1979. Sémiologie générale de l'effort et de la fatigue chez les animaux de sport. Bull. Soc. Sci. Vet. Méd. Comparée, 81, 17-32.

Larvor P., Violette C., 1969. Influence de l'ingestion d'herbe tétanigène sur le métabolisme minéral ( $\mathrm{Mg}, \mathrm{Ca}, \mathrm{P}, \mathrm{Na}, \mathrm{K}$ ) et certains éléments du métabolisme énergétique (corps cétoniques, acides gras volatils) chez la brebis. Nouvelle hypothèse pathogénique sur la tétanie d'élevage. Rech. Vét., 2, 27-44.

Larvor P., Rayssiguier Y., 1978. La tétanie d'herbage. Le Point Vétérinaire, 7 (31), 79-86.

Lellouch J., LAZAR P., 1974. Méthodes statistiques en expérimentation biologique. Flammarion Médecine Sciences, Paris, 283 p.

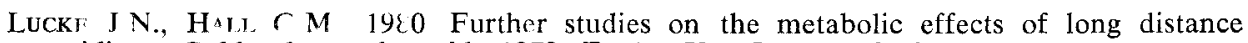
riding: Golden horse shoe ride 1979. Equine Vet. J., 12, 189-192.

MARION J.M., 1984. Magnésium et effort d'endurance chez le poney. DEA Nutrition, Paris VI, $48 \mathrm{p}$.

MeYer H., 1979. Magnesium Stoffwecksel und Magnesium bedarf Pferdes. Ubers. Tierernähr. 7. $75-92$. 
National Research Council (N.R.C.), 1978. Nutrient requirements of domestic animals $\mathrm{n}^{*} 6$ (National Academy of Sciences). Nutrient Requirements of Horses, 4th Edition, Washington D.C., $33 \mathrm{p}$.

Nehring K., Schiemann R., Hoffman L., Jentsch W., 1963. Die Energetische Verwertung der Futterstoffe. Arch. Tierernähr., 13 (3), 119-132.

RAYSSIGUieR Y., 1977. Hypomagnesemia resulting from adrenaline infusion in ewes. Its relation to lipolysis. Horm. Met. Res., 9 (4), 309-314.

RaYssiguier Y., 1981. Magnesium and lipids interrelationships in the pathogenesis of vascular disease. Magnesium Bull. 1a, 165-177.

Rayssiguier Y., LARvor P., 1976. Hypomagnesia following stimulation and lipolysis in ewes. Effect of cold exposure and fasting. In : Magnesium and disease. 2nd International Symposium on Magnesium. Montréal, 30 mai-1 $^{\text {er }}$ juin. Spectrum Publications Inc. (1980), 67-72.

Ricaux S., 1982. Etude de la digestibilité de la paille sodée, du gluten-feed et du papier chez le poney. Rapport de stage BTS, Centre National de promotion rurale. ClermontFerrand, $105 \mathrm{p}$.

Schiemann R., Nehring K., Hoffmann L., Jentsch W., Chudy A., 1971. Energetische Futterbewertung und Energienormen - VEB DT Landw. Verlag, Berlin, 344 p.

Schwartz D., 1969. Méthodes statistiques à l'usage des médecins et des biologistes. Flammarion. Médecine Sciences, Paris, 318 p.

Snow D.H., Kerr M.G., Nimmo A., Aвbott E.A., 1982. Alterations in blood, sweat, urine and muscle composition during prolonged exercice in the horse. Vet. Rec., 10, 377-384.

VAlETte J.P., 1984. Nutrition lipidique et aptitudes à l'effort d'endurance chez le poney. Thèse Doctorat Nutrition Université, Paris VI, $84 \mathrm{p}$.

Walsh J.J., Harrington D.D., 1973. Availability of $\mathrm{MgO}, \mathrm{MgSO}_{4}$ and $\mathrm{MgCO}_{3}$ in Equine. J. Anim. Sci., 35, 206 (Abstr.).

Wolter R., Valette J.P., Durix A., Letourneau J.C., Carcelen M., 1982. Digestibilité comparée de quatre céréales (avoine, orge, maïs, blé) selon le mode de présentation chez le poney. Ann. Zootech., 31, 445-458.

Wolter R., Grandjean D., ValetTe J.P., 1984. Relations nutrition - aptitudes sportives méthodologie de l'étude expérimentale chez l'animal en effort d'endurance. Rec. Méd. Vét., $180(7-8), 657-668$.

Wolter R., Valette J.P., 1986. Digestibilité de régimes hyperlipidiques chez le poney. Reprod. Nutr. Dévelop., 23, (1 B), 337-338. 